\section{Jahre Viktimologie-Sommerkurs in Dubrovnik}

Otmar Hagemann, Peter Schäfer und Stephanie Schmidt (Hrsg.): Victimology, Victim Assistance and Criminal Justice - Perspectives Shared by International Experts at the Inter-University Centre of Dubrovnik. Mönchengladbach: Fachhochschule Niederrhein 2009, 304 S., $16,50 €$.

Der von Otmar Hagemann, Peter Schäfer und Stephanie Schmidt herausgegebene Band „Victimology, Victim Assistance and Criminal Justice“ enthält seinem Untertitel entsprechend Ansichten internationaler Experten der Viktimologie, die im Sommerkurs am InterUniversity Centre in Dubrovnik lehrten und lehren. Das Buch ist zugleich eine Festgabe zum 25. Geburtstag des Kurses.

In seiner Einleitung führt Otmar Hagemann nicht nur die einzelnen Beiträge ein, sondern formuliert auch den Anspruch des Buches: Es soll kein umfassender Überblick über den Stand der viktimologischen Forschung gegeben werden, sondern der Band soll durch die Bandbreite der Beiträge das Interesse insbesondere von Studierenden an der Viktimologie wecken.

Konsequenterweise folgt mit dem Beitrag von Jo-Anne Wemmers eine kurze und knappe Übersicht über die Entwicklung der Viktimologie, in der vor allem die Bedeutungen des Wortes „Opfer“, die Anfänge der Viktimologie in der Nachkriegszeit und die Institutionalisierung durch die World Society of Victimology beschrieben werden.

Stephan Parmentier untersucht die Aktivlegitimation bei Individualbeschwerden vor der früheren Europäischen Kommission des Europarats mit einem Schwerpunkt auf dem Opferbegriff der EMRK als Merkmal der Prozessführungsbefugnis. Einziges Manko dieser genauen Analyse ist, dass Parmentier sich auf die Rechtsprechung der Kommission beschränkt und nicht darauf eingeht, inwieweit sie nach der Reform des Verfahrens vom Gerichtshof fortgeführt wurde.

Stephanie Schmidt schreibt über Auswirkungen der Richtlinie 2008/52/EG des Europäischen Parlaments und des Rates vom 21. Mai 2008 über bestimmte Aspekte der Mediation in Zivil- und Handelssachen auf die Streitschlichtung und über den schon vor der Richtlinie vorgelegten niedersächsischen Entwurf eines Gesetzes über die Einführung eines Mediations- und Gütestellengesetzes (Nieders. Landtag, Drs. 15/3708), der nach ihrer Ansicht als Vorbild für ein entsprechendes Bundesgesetz dienen könne.

Nils Christies sehr kurzer Beitrag entspricht nicht dem üblichen $\mathrm{Mu}-$ ster eines wissenschaftlichen Aufsatzes, sondern dokumentiert einen stream of consciousness zur Opferrolle. Diese sei in unserer heutigen Gesellschaft, in der sich jeder seine Identität selbst basteln müsse, nicht nur eine mögliche, sondern eine eventuell auch attraktive Identität - anders als zu nicht näher bestimmten früheren Zeiten, als Rollen und Identitäten durch Tradition vorgegeben gewesen seien. Mit der Opferrolle seien jedoch viele Gefahren verbunden, die die Entwicklung zu einem gesunden und selbstbestimmten Menschen behinderten. Die Bedenken, die Christie äußert, sind wichtig und hätten eine vertiefte wissenschaftliche Auseinandersetzung verdient, allerdings hätte dies dann nicht in Form dieses Zwischenrufs geschehen können.

Lode Walgrave analysiert die Rolle des Opfers im Restorative Justice-Ansatz. Er setzt sich mit typischen Einwänden gegen diesen Ansatz aus der Sicht von Opfern einer Straftat auseinander und präsentiert empirische Befunde zur Beteiligung von Opfern an Restorative Justice-Angeboten sowie zu ihrer Zufriedenheit mit dem Prozess und seinen Ergebnissen. Er schließt mit dem Hinweis, dass Opfer von Straftaten mit Restorative Justice viel gewinnen könnten, dass es jedoch nicht ausreiche, wenn entsprechende Elemente an den Rändern des traditionellen Strafjustizsystems existierten, vielmehr müsse das System selbst umstrukturiert werden. Diese Forderung mag man angesichts der Beharrungskräfte innerhalb der deutschen Strafjustiz für naiv halten, allerdings wurden in das Jugendkriminalrecht von Walgraves Heimatland Belgien vor allem aufgrund seines Einflusses Strukturen der Restorative Justice eingeführt (vgl. Christiaens/Dumortier/Nuytiens, in: Dünkel u. a. (Hrsg., 2010): Juvenile Justice Systems in Europe).

Bernard Jervis berichtet über seine Forschung über die Verarbeitung eines serbischen Angriffs auf die bosnische Stadt Tuzla im Mai 1995 durch Opfer, deren Angehörige und die örtliche Gemeinschaft. Die meisten Todesopfer, die allen drei Bevölkerungsgruppen der Stadt - Serben, Kroaten und bosnischen Muslimen - angehörten, wurden gemeinsam auf einem dafür eingerichteten, zentral gelegenen Friedhof bestattet. In einer qualitativen Studie untersuchte Jervis, welche Auswirkungen das Erlebnis auf die Angehörigen der Toten und auf die Überlebenden hatte und welche Bedeutung die gemeinsame Begräbnisstätte für sie und die Gemeinde insgesamt hat. Die Bearbeitung dieses interessanten Themas hätte noch gewonnen, wenn Jervis sein methodisches Vorgehen genauer beschrieben hätte. Zudem wäre es interessant gewesen, die näheren Umstände der Planung des gemeinsamen Begräbnisses genauer zu analysieren, die noch während des Krieges unter dem unmittelbaren Eindruck des Angriffs stattgefunden haben muss - das genaue Datum nennt er nicht.

Christine Alder und Kenneth Polk schreiben über das zunächst exotisch anmutende, sehr interessante Thema des illegalen Handels mit Antiquitäten und archäologischen Artefakten aus viktimologischer Sicht und erläutern Lösungsmöglichkeiten unter Einbeziehung des Restorative Justice-Ansatzes. Sie stellen fest, dass traditionelle Bekämpfungsstrategien des Kriminalrechts nicht effektiv sind, sondern durch das Verbot des Handels und der Ausfuhr in den Herkunftsländern weitere Kriminalität (Bestechung, Schmuggel) nach sich ziehen. Sie schlagen vor, statt allein auf Bestrafung auf Überzeugung zu setzen und beschreiben als prototypischen Fall den Umgang mit einem Schiffsfund in Vietnam, bei dem das mögliche Opfer - der Staat, dessen kulturelles Erbe angegriffen wird - mit den möglichen Tätern - Bergungsunternehmen, Zwischenhändler, Galeristen in den Abnehmerländern - eine kontrollierte Einführung einzelner Funde in den Markt nach der wissenschaftlichen Auswertung aushandelte.

Hans-Jürgen Kerner fasst in seinem Beitrag Erkenntnisse zur Täterund Opferschaft junger Menschen zusammen. Wie er selbst schreibt, sind diese Befunde der wissenschaftlichen Gemeinschaft bekannt. Bei anderen Bevölkerungskreisen - auch Studierenden - kann man dieses Wissen aber anscheinend nicht voraussetzen, wie die Reaktionen auf den sog. Münchner U-Bahnfall vom Dezember 2008 zeigen, die Kerner eingangs rekapituliert. Zur Illustration dienen hier Daten aus der Schülerbefragung des KfN von 2007/2008, PKS-Daten von 2007 und Daten der U. S. National Youth Survey, einer repräsentativen Befragung junger US-Amerikaner.

Robert Peacock referiert Ergebnisse seiner Forschung über Viktimisierung von jugendlichen und heranwachsenden Gefangenen im südafrikanischen Strafvollzug. Die materiellen Bedingungen sind dort überaus ungünstig und bilden eine Grundlage für vielfältige soziale Abhängigkeiten und Gelegenheit für Unterdrückung. Peacock gliedert die Beschreibung in Aspekte der Gewalt durch die Institution (Unterbringung und Probleme mit dem Personal) und der Gewalt unter den Gefangenen (subkulturelle Regeln, Gangs, sexuelle Gewalt) und hält fest, dass es derzeit im südafrikanischen Strafvollzug keine nennenswerten Präventions- oder Behandlungsangebote gebe. 
Janice Josephs Beitrag behandelt Tötungsdelikte an Frauen in den USA unter besonderer Berücksichtigung schwarzer Opfer. Insgesamt sind schwarze Frauen gemessen an ihrem Anteil an der US-amerikanischen Bevölkerung unter den Opfern deutlich überrepräsentiert. Erklärungsansätze für das Ausmaß tödlicher Gewalt gegen schwarze Frauen macht Joseph in den Ursachen häuslicher Gewalt unter schwarzen US-Amerikanern aus, für die wiederum Aspekte von Sexismus, Rassismus und sozialem Status wesentlich seien. Es gebe Risikofaktoren auf der Ebene des Individuums, der Paarbeziehung, der Gemeinschaft sowie kulturelle Faktoren. Zur Prävention schlägt sie gemeinsame Angebote verschiedener Dienste unter Berücksichtigung kultureller Besonderheiten der afro-amerikanischen Gemeinde vor, ohne hier auf Einzelheiten einzugehen.

Sarah Ben-David stellt drei theoretische Erklärungsansätze für das Phänomen, dem Opfer Schuld an seinem Schicksal zu geben, vor. Als Beispiel dienen die Umstände, die die Zuschreibung von Schuld bei Vergewaltigungsopfern beeinflussen. Die bisher zur Diskussion gestellten Erklärungsansätze sind der Glaube an eine gerechte Welt, mit dem die Vorstellung unschuldiger Opfer nicht vereinbar ist, so dass zur Aufrechterhaltung des Glaubens den Opfern Schuld zugeschrieben werden muss, die Theorie der defensiven Attribution, nach der dem Opfer Schuld zugeschrieben wird, um sich selbst zu vergewissern, dass einem so etwas nicht passieren kann, sowie das kontrafaktische Denken, ein kognitiver Mechanismus, durch den z. B. das Verhalten des Opfers vor der Tat so transformiert wird, dass das Ergebnis sich ändert („,wäre sie nicht mit ihm gefahren, hätte er sie nicht vergewaltigt“"). Ben-David schließt damit, dass mit der Theorie der defensiven Attribution am besten vorhergesagt werden kann, wer einem Opfer Schuld zuschreiben wird. Welchem Opfer unabhängig vom Beobachter - am ehesten die Schuld gegeben wird, lässt sich jedoch mit keiner der Theorien gut vorhersagen.

Ute I. Haas schreibt über die Unterstützung von Opfern häuslicher Gewalt in Deutschland. Sie beschreibt die Entwicklung bis zum Gewaltschutzgesetz 2002 sowie den Inhalt des Gesetzes selbst, die entsprechenden Ergänzungen im Polizeirecht der Bundesländer erwähnt sie nicht. Außerdem geht sie auf die Bemühungen des Bundeslandes Niedersachsen um einen gemeinsamen pro-aktiven Ansatz von Polizei, Frauenhäusern und Beratungsstellen ein, die es übrigens ähnlich auch in anderen Bundesländern gibt. Darüber hinaus berichtet sie über die besonderen Probleme von Kindern misshandelter Mütter, über die Ergebnisse der Untersuchung des Bundesfamilienministeriums über weibliche Opfer häuslicher Gewalt (Lebenssituationen, Sicherheit und Gesundheit von Frauen, 2004) sowie Probleme bei der Beschreibung von Erfolgskriterien von Arbeit mit dieser Gruppe.

Natti Ronel beschreibt eine von ihm selbst entwickelte spirituelle Therapie für Opfer, die er als Grace Therapy bezeichnet und die auf dem 12-Schritte-Modell basiert. Ausgangspunkt ist die Erfahrung der Machtlosigkeit anlässlich der Viktimisierung, zum einen während des Geschehens selbst und zum anderen aufgrund des Einflusses der Viktimisierung auf das weitere Leben. In der Therapie geht es darum, die erste Erfahrung der Machtlosigkeit zu akzeptieren, da sie nicht mehr geändert werden kann und im Hinblick auf die zweite pragmatisch zu ergründen, welche Umstände geändert werden können, um sich aus der Machtlosigkeit zu befreien und dies dann auch zu tun.

Peter Schäfer nimmt die Idee einer Mediation bei hochstrittigen Scheidungen und Trennungen zum Anlass, diese alternative Konfliktlösungsmöglichkeit vorzustellen und den typischen Ablauf zu beschreiben. In den USA seien Eltern minderjähriger Kinder verpflichtet, im Scheidungsverfahren eine Mediation zu unternehmen. Schäfer hält dies auch bei hochstrittigen Trennungen für eine vielver- sprechende Möglichkeit, da sich die Eltern eventuell eher als in einem gerichtlichen Scheidungsverfahren ihrer Verantwortung für die Kinder bewusst würden und zu einem konstruktiven Umgang mit einander finden könnten.

Ivo Aertsen und Frank Hutsebaut berichten Ergebnisse eines eigenen Forschungsprojekts in Belgien über Eltern, die ein Kind in einem Verkehrsunfall verloren haben. Untersucht wurden Bedürfnisse und Erfahrungen der Angehörigen getöteter Kinder, die Berücksichtigung dieser Bedürfnisse in den allgemeinen Opferschutzgesetzen und die Wirksamkeit der Unterstützung durch spezialisierte Beratungsstellen. Kern der Studie ist die qualitative Befragung betroffener Eltern. Hier wird deutlich, dass sie vor allem das Bedürfnis nach umfassender und rascher Information über das unmittelbare Geschehen, nach einer zügigen Abwicklung der polizeilichen und gerichtlichen Untersuchung sowie einer angemessenen Beteiligung im gesamten Prozess haben.

Albert R. Hauber beschreibt die drei niederländischen Projekte Halt, ATAS (Amsterdam Tourist Assistance Service) und Stadswacht (Stadtwächter), die im weitesten Sinne auf die Reduzierung von Viktimisierung abheben und über die er als Lehrer des Sommerkurses berichtet hat.

Otmar Hagemanns Artikel behandelt mit den Gemeinschaftskonferenzen in Elmshorn den ersten Versuch, in Deutschland Family Group Conferencing zu etablieren. Dies ist eine Mediationsform, an der anders als beim Täter-Opfer-Ausgleich nicht nur Täter, Opfer und ein neutraler Dritter beteiligt sind, sondern auch Familienmitglieder, Freunde und andere wichtige Personen. Erste Erfahrungen aus fünf Konferenzen zeigen, dass die Beteiligung von Unterstützern insb. auch für die Täter sehr wichtig ist, da sie allein mit der Situation häufig überfordert sind.

L. Thomas Winfree schreibt über Restorative Policing als Konzept der Polizeiarbeit innerhalb eines übergeordneten Restorative JusticeAnsatzes in der Strafverfolgung und geht vor allem auf Probleme durch gegensätzliche Konfliktlösungsstrategien und -ziele in der europäisch-nordamerikanischen Kultur und in Kulturen von Ureinwohnern ein. In den USA werde die Umsetzung dieses Konzepts aber auch durch Besonderheiten der Organisation der Polizei und ihres Selbstverständnisses sowie durch gesellschaftliche Rahmenbedingungen, die sich auf die Rolle der Polizei auswirken würden, behindert.

Zum Abschluss erzählt Xin Ren über die prägenden Erfahrungen des Sommerkurses in Dubrovnik und die wichtige Rolle der Menschen, die sie dort kennengelernt hat, für ihr Leben als Wissenschaftlerin.

Das Buch bietet eine kurzweilige Mischung an Beiträgen zu Themen, die in der Viktimologie wichtig sind. Sprachliche Ungenauigkeiten waren, wie Otmar Hagemann in der Einleitung schreibt, bei diesem englischen Werk angesichts der in der Mehrzahl nicht muttersprachlichen Beteiligten sowie der knappen Zeit erwartbar, sind aber zu verschmerzen. Dieser Band ist insgesamt sehr interessant und scheint mir aufgrund der Kombination aus Wissensvermittlung und aktueller internationaler Forschung geeignet, gerade auch bei Studierenden Neugier auf viktimologische Fragestellungen zu wecken. Das Werk wird also seinem Anspruch gerecht.

Dr. Kirstin Drenkhahn ist wissenschaftliche Mitarbeiterin am Lehrstubl für Strafrecht und Strafverfahrensrecht (Prof. Dr. C. Sowada) und am Lehrstubl für Kriminologie (Prof. Dr. F. Dünkel) an der Universität Greifswald. Sie hat vor einigen Jahren am Sommerkurs in Dubrovnik teilgenommen. 